\title{
ALONSO DE CASTILLO SOLÓRZANO REESCRITOR DE SÍ MISMO: ALGUNAS NOTAS SOBRE LOS ESCARMIENTOS DE AMOR MORALIZADOS Y EL LISARDO ENAMORADO ${ }^{1}$
}

\author{
Givlia Giorgi \\ Università degli Studi di Ferrara \\ giulia.giorgi@unife.it
}

L

a producción literaria de Alonso de Castillo Solórzano - y sobre todo su narrativa — ha sido objeto de muchas críticas por sus límites estilísticos, la falta de originalidad y el escaso interés de las vicisitudes narradas ${ }^{2}$. Se le ha considerado - y no sin razón — un simple profesional de la escritura al servicio de un público cortesano hambriento de historias amorosas, casos curiosos y aventuras; un autor, pues, que se muestra como perfectamente alineado con el género de literatura — por así decir “comercial”- que se leía en aquella época ${ }^{3}$. Otro defecto que se le imputa a menudo es la pasiva imitación — pese a la incesante defensa de la originalidad de su obra — de los novellieri y de la tradición novelística italiana, no solo por lo que atañe a elementos estructurales (como el marco que incluye sus narraciones breves), sino también a estilemas, topoi e incluso historias

1 Este trabajo se inscribe en el marco del Proyecto I+D+i del MINECO La novela corta del siglo XVII (y II) (FFI2013-41264-P).

2 La crítica moderna ha reevaluado el juicio sobre el autor vallisoletano —alabado por sus contemporáneos y muy exitoso en su época-, y sobre todo a partir de los años 50 del siglo pasado, se ha vuelto particularmente severa. Más que dura resulta la opinión de Dunn (1952), autor de la primera monografía sobre el vallisoletano, quien describe a Castillo como un claro ejemplo de la decadencia de la narrativa barroca; por su parte, Velasco Kindelán (1983) lo define como un escritor poco original; en particular: "Castillo es un hábil componedor de historias de "honesto entretenimiento", a base de unos materiales que no son otros que los que le vienen dados por la tradición literaria y algunos escritores contemporáneos suyos [...]. Pero sobre todo por los consensos comunes, las ideologías vigentes, los criterios aceptados, aquello en lo que “todos” están de acuerdo. Castillo es un espíritu conservador, transmisor de un consenso común» (1983: 17).

3 Sobre la fortuna editorial de la narrativa española del Siglo de Oro se remite a Whinnom (1980), Pacheco-Ransanz (1986) y Fernández Insuela (1993). 
enteras ${ }^{4}$; asimismo, se ha demostrado el importante influjo, para la redacción de sus novelas, del teatro nacional de su tiempo ${ }^{5}$.

En otra ocasión, analizamos las técnicas empleadas por Castillo a la hora de reelaborar los textos de los novelistas italianos para adaptarlos a un contexto cultural tan diferente como el de la España contrarreformista, revelando que las estrategias "moralizantes" preveían la interpolación de comentarios del narrador que estigmatizasen los comportamientos considerados intolerables por aquel entonces, la ampliación de las historias (con la inserción de acciones y personajes secundarios) y la completa supresión de alusiones sexuales inoportunas ${ }^{6}$.

En este trabajo, en cambio, queremos estudiar qué ocurre cuando un autor como Castillo Solórzano reescribe su propia obra. No estamos aludiendo a las reelaboraciones dramáticas de novelas cortas — como Los encantos de Bretaña (Fiestas de jardín, 1634), dramatización de La cautela sin efecto (Noches de placer, 1631) ${ }^{7}$ - sino de una segunda edición (retocada) del mismo texto: la novela sentimental Escarmientos de amor moralizados (Sevilla, 1628) se editó nuevamente, un año más tarde, con otro título: Lisardo enamorado (Valencia, 1629). ¿Qué acaeció durante aquellos pocos meses? ¿Por qué publicar el mismo texto, si bien con numerosas intervenciones estilísticas y algunos cambios significativos, a tan corta distancia? Serán estas algunas de las preguntas a las cuales se procurará responder durante la edición del Lisardo enamorado (en adelante, Lisardo) que afrontaremos en los próximos dos años. De momento, nos limitaremos a recoger los (exiguos) datos que se coligen de los preliminares de los dos volúmenes y a comparar el primer capítulo de ambas novelas, para mostrar de qué modo el autor refundó los Escarmientos de amor moralizados (en adelante, Escarmientos).

Los Escarmientos se publicaron en 1628 en la imprenta sevillana de Manuel Sande ${ }^{8}$; el mismo año, el marqués de los Vélez —a quien se dedica el volumen y también su reedición - fue nombrado virrey de Valencia y parece sensato conjeturar que Castillo Solórzano, maestresala de su casa, residiese en la ciudad del Turia junto con él. No olvidemos, además, que en Valencia se estampó también otra obra solorzaniana: la Huerta de Valencia (1629)9. En opinión de Juliá

Véanse a este propósito Bourland (1927: 59-60), Dunn (1952: 20-23) y Giorgi (2013: 25-32).

5 Sobre la relación entre la novela corta y el género dramático en el Siglo de Oro, se remite a Morínigo (1957), Yudin (1968; 1969), Baquero Goyanes (1983), Gutiérrez Hermosa (1997, pp. 167-174) y Miñana (1998). Para el influjo del teatro nacional en la producción narrativa de Castillo, véanse Sileri (2008) y Giorgi (2013: 32-42).

6 Véase Giorgi (2012).

7 Para la novela y su adaptación teatral se remite a Sileri (2008: 220-245); la edición moderna de dicha comedia se debe a Bacchelli (Castillo Solórzano, 1980).

8 Las licencias y aprobaciones se firmaron, empero, en los meses de febrero y marzo del año precedente.

9 A la muerte del marqués, en diciembre de 1630, el autor pasó supuestamente a Barcelona, donde se dieron a la imprenta Noches de placer (1631), Las harpías en Madrid (1631), La niña de los 
Martínez, “editor” moderno del Lisardo, el autor acompañó al marqués en un viaje a Sevilla y allí dio a la imprenta los Escarmientos, pero su estancia en la capital andaluza debió de ser muy breve, pues ya en mayo de 1628 se firmaban en Valencia las aprobaciones del Lisardo. Más sugestiva resulta la conjetura de Dunn, según el cual la doble edición del texto se configuraría simplemente como una hábil operación de marketing. Centrándose en lo que más distingue a las dos ediciones - la falta, en el Lisardo, de los aprovechamientos morales que cierran cada capítulo-, Dunn escribe:

Castillo son realized how invalid these appendages [se refiere a los Aprovechamientos] were. After the Jornadas alegres he ceased to preface his stories with them, and in 1629 he republished the Escarmientos de amor stripped of the moralities attached to each chapter, with the new title of Lisardo enamorado. Were novels with ostentatiously moral titles losing popularity? Or was this just a trick to sell the same book twice? The Escarmientos were printed in Seville, the Lisardo in Valencia. It is possible that Castillo had the Lisardo ready in case the other should be a failure - at any rate he had the licencia for it three months before the tassa was made out for the Escarmientos. Had the Escarmientos been a success he would hardly have needed to produce the Lisardo (Dunn 1952: 77).

La diferencia más evidente entre los Escarmientos y el Lisardo, de hecho, es la completa supresión de los «Aprovechamientos», breves apartados en los que el autor resume lo que se acaba de contar observando, caso por caso, los comportamientos de los personajes y censurando los que resultan moralmente inaceptables. Otra vez, pues, topamos con la tan conocida — y deplorada — afición moralizadora de Castillo ${ }^{10}$. Pero, ¿por qué decidió eliminar tales escolios en el Lisardo? ¿Tal vez porque se dio cuenta de la inutilidad de esos recursos en un género de literatura destinado a un público cortesano más interesado en entretenerse con la lectura que en ver condenados sus propios hábitos? Coincido con Dunn en afirmar que los lectores de la época ya no querían este tipo de adoctrinamiento ${ }^{11}$ y se alejaban del «excesivo prurito moralista de [la] novela cortesana» (González de Amezúa 1929: 98), que acarrearía su inevitable decadencia. La verdad es que solo en otra novela, Las harpías en Madrid (1631), Castillo volvió a los «Aprovechamientos» después de cada estafa y, a partir de aquel momento, dejó «sus reflexiones para

embustes (1632) y Los amantes andaluces (1633), volviendo a Valencia hacia 1634. Según Juliá Martínez «allí debió de estar, hasta que, el año siguiente marchó a Aragón porque, terminado el tiempo del virreinato en Valencia, pasó a Zaragoza su mecenas don Pedro Fajardo de Zúñiga y Requesens, quinto Marqués de los Vélez, quien había sucedido a su padre en el cargo de la ciudad del Turia» (Juliá Martínez 1947: 18-19).

10 Véase Dunn (1952: 75-86).

11 «I am inclined to think that the public was beginning to tire of being told what moral it ought to draw from its entertainment» (Dunn, 1952: 77). 
que fuesen vislumbradas por el lector, o para ser expuestas en medio de la narración» (Juliá Martínez 1947: 28).

Cotejando las dos versiones del Lisardo, y concretamente el primer capítulo, se observa que, si bien las moralejas finales desaparecen, se conservan todavía los eventuales comentarios diseminados dentro del texto. Por ejemplo, en los Escarmientos $^{12}$ se lee:

una amiga de Gerarda envió a una criada que me dijese que unas damas me pedían que me llegase a su asiento, porque deseaban hablarme. Yo la respondí que en la iglesia era muy grosero en no obedecer tales mandatos, por parecerme que los templos se hicieron más para la oración que para hablar en cosas diferentes de esto. Y cierto, amigo —aunque sea paréntesis de mi narración—, que es la mayor lástima del mundo ver lo que de esto pasa en la corte, sin que haya remedio para quitar esta perniciosa costumbre tan introducida, que más parecen templos de gentiles en este particular que de cristianos con lumbre de fe. Pero esto remédienlo aquellos a quien toca, que no harán poco servicio a Dios y amistad a los que castigados escarmentaren. Vuelvo al hilo de mi historia y digo que negué el obedecerlas en la iglesia; pero dije que fuera de ella acudiría a donde me mandasen ir a besarles sus manos y que si importaba el no ser vista, yo tenía la casa de un amigo cerca, donde me podrían hacer merced (Escarmientos: f. 6r-v).

En el Lisardo ${ }^{13}$, amén de unos pocos cambios estilísticos y sintácticos, se mantiene la moral, si bien dislocada al final del fragmento:

Una ${ }^{14}$ amiga de Gerarda envió a una criada suya que de su parte, sin decir quién era, me dijese que unas damas deseaban que me llegase más cerca de ellas que deseaban hablarme. Yo la respondí que en las iglesias era muy grosero en no obedecer tales mandatos, por parecerme que los templos se hicieron más para la oración que para hablar en cosas ajenas de esto; que, si fuera de la iglesia gustaban que yo les besase sus manos y acudiese a lo que fuesen servidas de mandarme, me avisasen de su gusto y que, si importaba ser secreto, que yo tenía la casa de un amigo allí donde me podrían hacer merced. Y cierto, amigo, que — aunque sea paréntesis de mi discurso — es la mayor lástima del mundo ver lo que de esto pasa en la corte, sin que haya remedio para quitar esta perniciosa costumbre tan introducida, que más parecen templos de gentiles los que hay en ella que de cristianos con lumbre de fe;

12 Se cita a partir de la princeps de 1628: en la transcripción de los fragmentos textuales se retoca la acentuación y la puntuación y se normaliza el uso de las mayúsculas; se resuelven las abreviaturas y se restaura la consonante nasal abreviada con el titulus; se uniforman las variantes gráficas de un mismo fonema, como $\mathrm{b} / \mathrm{u} / \mathrm{v}, \mathrm{c} / \mathrm{ç} / \mathrm{z}, \mathrm{ss} / \mathrm{s}, \mathrm{g} / \mathrm{j} / \mathrm{x}, \mathrm{i} / \mathrm{y}$; se resuelven las aglutinaciones de la preposición «de» + pronombre o adjetivo demostrativo y «de» + pronombre personal («de esta» en lugar de «desta», «de él» en lugar de «dél», etc.).

13 Se cita la princeps de 1629. En la transcripción se siguen los criterios definidos en la nota 12.

14 Se corrige la princeps que leía «A una». 
pero esto remédienlo aquellos a quien toca, que no harán poco servicio a Dios y amistad a los que, castigados, escarmentaren (Lisardo: 13-14).

Y poco después, volviendo al mismo tema, añade: «Gerarda aprobó por buena mi respuesta y le pareció acertada la consideración de no querer dar escándalo en el templo con pláticas ociosas, siendo edificado y constituido para solo alabar a Dios» (Escarmientos: 6v); tampoco este comentario desaparece en el Lisardo, donde además se refina: «mi Gerarda aprobó por buena mi respuesta y le pareció acertada la consideración de no querer profanar con pláticas ociosas el sagrado lugar dedicado para solo alabar a Dios» (Lisardo: 14).

Sigamos nuestro análisis de las diferencias entre una y otra versión de la obra y comparemos los prólogos. En los Escarmientos el autor defiende el tono mediano de su escritura, alejándose de todo estilo culto:

En este corto volumen te ofrezco un libro en lengua castellana, como la hablaron mis padres y abuelos, no transformada en varios disfraces por no verla en varias fortunas; no escrita en Carnestolendas, donde el tizne de lo inculto la haga tan obscura como desconocida; no desfigurada entre la harina y salvado (Escarmientos: f. 7v).

Reitera este concepto en el Lisardo, donde, persiguiendo aún mayor claridad, afirma:

su estilo no es tan cuidadoso que se acoja a esto que llaman culto, ni tan relevante que le ignore por escuro el que le desea entender, porque no quiero que este libro se compre por no inteligible, que estuviera a peligro de correr varias fortunas, hallando en él ignorancias apiñadas; su lenguaje es claro y, si humilde, con él han corrido otros de su mismo autor por manos de quien les ha honrado (Lisardo: f. 4r).

Y además, en esta segunda edición Castillo parece justificarse sobre el contenido real del volumen - ¿habían aflorado las críticas después de aparecer los Escarmientos?-; el autor escribe: «una novela te presento, temeroso de lo que te ha de parecer, pues va preñada de muchas» (Lisardo: f. 4r). En efecto, el Lisardo (y su precedente) sigue el camino de su homónimo protagonista y dedica cada capítulo a la historia de uno de los personajes que el caballero encuentra durante su viaje: por esta razón, se parece más a una colección de novelas que a una novela unitaria ${ }^{15}$.

Para terminar este breve y parcial estudio sobre los Escarmientos y el Lisardo, pasemos a estudiar algunas de las modalidades de reescritura aplicadas por Castillo

15 No es prerrogativa exclusivamente solorzaniana la de intercalar novelas dentro de textos más amplios, sino una costumbre bastante común en la literatura aurisecular. Sobre las digresiones y los relatos intercalados, véase, entre otros, Hernández Varcárcel (1980), Brau (1991) e Yllera (1992). 
en el trasvase de un texto a otro. Por tratarse de un trabajo todavía in fieri - $-\mathrm{y}$ preparatorio para una sucesiva edición- decidimos centrarnos tan solo en el primer capítulo, si bien las variantes parecen intensificarse conforme avanza la obra ${ }^{16}$. Simplificando, se podrían distinguir cuatro géneros de intervención que, como veremos, responden a necesidades muy distintas y, a veces, en aparente contraste.

\section{INTERPOLACIÓN DE ADJETIVOS}

Uno de los elementos característicos del estilo solorzaniano es, según la crítica, su verbosidad y la redundancia adjetival. En opinión de Dunn (1952: 65-66), son cuatro los tipos de «gratuitous adjective» que se hallan en el corpus narrativo de Castillo : 1) redundantes y tautológicos, como «verde yerba» 0 «roja sangre»; 2) estereotipados, como «apacible céfiro» o «gruesa encina»; 3) innecesarios, como el adjetivo «hermosa» continuamente aplicado a cada dama que aparece en la narración; 4) reduplicados, como la noche que es a menudo «oscura y tenebrosa», o el invierno «encogido y erizado». En la reelaboración de los Escarmientos, Castillo añade nuevos - y poco útiles — adjetivos en muchos de los fragmentos retocados. Por ejemplo:

a) «verdugos de mi alma» (Escarmientos: f. 1r) se convierte en «verdugos crueles de mi alma» (Lisardo: 1);

b) «nuestra correspondencia» (Escarmientos: f. 3r) se transforma en «nuestra frecuentada correspondencia» (Lisardo: 5);

c) las «madres» (Escarmientos: f. 6r) de las damas son, por supuesto, «ancianas madres» en el Lisardo (13);

d) «mi venida» (Escarmientos: f. 12v) se convierte en «mi no esperada venida» (Lisardo: 30);

e) a «manifestaba su amor» (Escarmientos: f. 13r) se añade el adjetivo «encubierto» («manifestaba su encubierto amor», Lisardo: 30);

f) el «regocijo de toros» (Escarmientos: f. 19r) que se organiza en ocasión de una fiesta se vuelve «solemne» en el Lisardo (44).

II. SUSTITUCIÓN DE UN VOCABLO POR OTRO EN POS DE UNA MAYOR PRECISIÓN LÉXICA - O COMO VARIATIO - Y DE UNA EXPRESIÓN POR OTRA EQUIVALENTE.

En algunos casos, Castillo parece buscar un término más apropiado al contexto o sustituye simplemente una palabra con su sinónimo. Por ejemplo, describiendo

16 Confróntese Juliá Martínez (1947: 49). Recordemos, además, que los Escarmientos se dividían en siete libros, mientras que el material narrativo del Lisardo queda distribuido en ocho. 
la relación entre dos damas que intervienen en el primer capítulo, habla antes de «estrecha amistad» (Escarmientos: f. 10v) y luego de «apretada correspondencia» (Lisardo: 24); en otra ocasión, modifica la expresión «dijo con cautela» (Escarmientos: f. 11r) por «la dijo muy falsa» (Lisardo: 25), evidenciando aún más la astucia de la dama en burlar a su amiga Gerarda. Conservando el mismo sentido, el autor modifica la frase «me acuerdo bien de ellas» (Escarmientos: f. 8v) con «no he perdido de mi memoria» (Lisardo: 19), y también «apenas se divisaban los bultos de las personas» (Escarmientos: f. 23v), que se convierte en «apenas se conocían los bultos de la gente» (Lisardo: 54). En otros fragmentos, Castillo interviene tan solo reemplazando un vocablo por otro, como por ejemplo el adjetivo «contento» por «ufano», «esquiva» por «huraña»; o sustituye el verbo «hacerme merced» por «favorecerme», «entretener» por «divertir», «aventajarme» por «excederme», etc.

III. REELABORACIÓN DE PERÍODOS ENTEROS Y REVISIÓN DE LA SINTAXIS A MANERA DE EXPOLITIO

La tentativa de limpiar la sintaxis, eliminando prolijidades y simplificando las frases, se percibe en varios fragmentos. Por ejemplo, en los Escarmientos (f. 13r) se lee: «comenzó a suspirar, sentándose en una silla, todavía con el papel en las manos. Admirose mi prima de verla y al mismo paso se holgó de que hubiese surtido efecto nuestra traza», párrafo que se refina y abrevia en el Lisardo (30-31): «comenzó a suspirar sentándose en una silla, con el papel y el retrato en la mano, dejando admirada a mi prima cuanto contenta de esta acción que veía». Poco después, Castillo reescribe la poco elegante frase «Hízose mi prima de la que no lo entendía y preguntole que a qué fin decía aquello» (Escarmientos: f. 13r) de una manera más inteligible: «No se dio mi prima por entendida de la razón, y preguntola que a qué fin decía aquello» (Lisardo: 31). También otros períodos se muestran mucho más fluidos después de la revisión: «la verdad era que yo la quería sumamente y andaba fuera de mí viendo cuán severamente me había tratado. Y asimismo por temer favoreciese a otro galán» (Escarmientos: f. 18r) se trueca en «la verdad era que yo la quería entrañablemente y andaba fuera de mí viendo cuán severa se me mostraba, temeroso que favoreciese a otro galán» (Lisardo: 42-43); «tuve ocasión de pedir a mi dama perdón de la estratagema que con ella se había hecho y de besarla una blanca mano con gusto suyo» (Escarmientos: f. 18v) se convierte en «tuve ocasión de pedir a mi dama perdón de la estratagema que había usado, el cual alcancé de ella y en muestra de que era así le besé una de sus blancas y hermosas manos, con gusto suyo» (Lisardo: 43). 


\section{REDUNDANCIAS Y GUIÑOS AL ESTILO CULTO}

A diferencia de lo que acabamos de evidenciar — el refinamiento de las cláusulas para perseguir mayor claridad sintáctica — hay fragmentos del primer capítulo en las que el autor dilata el discurso intercalando informaciones innecesarias o repetitivas. Por ejemplo, cuando nombra a uno de los personajes, Castillo escribe: «Negrete, su fiel criado» (Escarmientos: f. 1v); y luego, «Negrete, que así se llamaba su fiel criado» (Lisardo: 2), utilizando la muletilla «así se llamaba», muy característica de su estilo. Inútil resulta también la aclaración («donde habían comido») que se añade en el Lisardo durante el siguiente párrafo: «siéndoles servida la comida [...], mientras los criados de don Félix y Lisardo comían, se quedaron los dos amigos en el aposento» (Escarmientos: f. 2v), que en la segunda versión se trueca en «siéndoles servida la comida [...], en tanto que los criados de don Félix y Lisardo comían, se quedaron los dos amigos en el aposento donde habían comido» (Lisardo: 5). Otras transformaciones resultan ampulosas y amaneradas — pero son muy típicas de la producción solorzaniana y, más en general, de la literatura de aquel tiempo-: con pocas palabras, don Félix cuenta cómo procuró informarse sobre las calidades de la dueña que amaba («supe la calidad de esta dama», Escarmientos: f. 5v); en el Lisardo, en cambio, dice: «traté luego de saber la calidad de quien había ya elegido por dueño de mi alma» (Lisardo: 12). Otro caso parecido es la sustitución del verbo «dormía» (Escarmientos: f. 2r) con «daba tributo a Morfeo» (Lisardo: 3).

En conclusión, este trabajo solo pretende mostrar de qué manera Castillo Solórzano intervino en los Escarmientos para editarlos nuevamente, apenas un año después, con el título de Lisardo enamorado. A partir de las variantes - exiguas pero significativas - que se han presentado no parece evidenciarse una estrategia de reescritura metódica y consciente encaminada a un objetivo específico (como alcanzar mayor claridad o enriquecer la prosa con un léxico florido, etc.), sino una remodelación del texto que, como vimos, responde a instancias muy diferentes entre sí. Es indiscutible que un análisis más amplio y profundizado de las diferencias textuales entre ambas novelas proporcionará otros datos sobre el proceso de reelaboración de la obra, sin duda valiosos para culminar su edición crítica y, quizá, solucionar eventuales loci critici.

Recibido: $29 / 08 / 2014$

Aceptado: 30/10/2014 


\section{OBRAS CITADAS}

Baquero Goyanes, Mariano (1983). «Comedia y novela en el siglo Xvil». En Serta Philologica F. Lázaro Carreter. Madrid: Cátedra, vol. II, pp. 13-29.

Bourland, Caroline B. (1927). The Short Story in Spain in the Seventeeth Century (with a Bibliography of the Novela from 1576 to 1700). Northampton: Smith College.

BraU, Jean-Louis (1991). Fonctions des nouvelles intercalées dans le roman spagnol au Siècle d'Or. Niza: Université de Nice.

Castillo Solórzano, Alonso de (1980). Los encantos de Bretaña. Franco Bacchelli (ed.). Verona: Università di Verona.

(1947). Lisardo enamorado. Eduardo Juliá Martínez (ed.). Madrid: Gráficas Ultra. (2013). Noches de placer. G. Giorgi (ed.). Madrid: Sial Ediciones.

Dunn, Peter N. (1952). Castillo Solórzano and the Decline of Spanish Novel. Oxford: Basil Blackwell.

FERNÁNDEZ Insuela, Antonio (1993). «Sobre la narrativa española de la Edad de Oro y sus reediciones en el siglo XVIII». Revista de Literatura 55, 109, pp. 55-84.

Giorgi, Giulia (2012). «“Novelar muy a imitación de lo de italia”: Castillo Solórzano, lector de Francesco Sansovino». En Rafael Bonilla Cerezo, José Ramón Trujillo y Begoña Rodríguez (eds.). Novela corta y teatro en el Barroco español (16131685). Studia in honorem Prof. Anthony Close. Madrid: Sial Ediciones, pp. 77-85. (2013). «Introducción», en A. de Castillo Solórzano. Noches de placer. Giulia Giorgi (ed.). Madrid: Sial Ediciones, pp. 9-68.

GonZÁlez de AmezúA, Agustín (1929). «Formación y elementos de la novela cortesana», Discurso de recepción pública en la Real Academia Española. Madrid:Tip. de Archivos.

Gutiérrez Hermosa, Luisa María (1997). «La constitución de un arte nuevo de hacer novelas». Exemplaria. Revista Internacional de Literatura comparada, 1, pp. 157-177.

Hernández VARCÁRCel, María del Carmen (1980). «El arte de la digresión y la voz del narrador en las Novelas a Marcia Leonarda». Anales de la Universidad de Murcia, 37, pp. 263-283.

Juliá Martínez, Eduardo (1947). «Observaciones preliminares». En Alonso de Castillo Solórzano, Lisardo enamorado. Madrid: Real Academia Española, Biblioteca Selecta de Clásicos Españoles, pp. 7-52.

MiÑAnA, Rogelio (1998). «La novela en escena: aspectos de la influencia del teatro sobre la novela corta del siglo XVII». En Edward H. Friedman, H. J. Manzari y Donald D. Miller (eds.), A Society on Stage. Essays on Spanish Golden Drama. Nueva Orleans: UP of the South, pp. 155-164.

Morínigo, Marcos A. (1957). «El teatro como sustituto de la novela en el Siglo de Oro». Revista de la Universidad de Buenos Aires, 2, pp. 41-61.

Pacheco-Ransanz, Arsenio (1986). «Varia fortuna de la novela corta en el siglo XviI». Revista Canadiense de Estudios Hispánicos, 10, 3, pp. 407-421.

Sileri, Manuela (2008). Le novelas cortas di Alonso de Castillo Solórzano tra narrativa e teatro [tesi dottorale]. Pisa: Università degli Studi di Pisa.

Velasco Kindelán, Magdalena (1983). La novela cortesana y picaresca de Castillo Solórzano. Valladolid: Institución Cultural Simancas. 
Whinnom, Keith (1980). "The Problem of the 'Best-Seller' in Spanish Golden-Age Literature». Bulletin of Hispanic Studies, 57, pp. 189-198.

YllerA, Alicia (1992). «El relato intercalado en la novela del XVII: ¿bello adorno o digresión enojosa?». En El relato intercalado. Madrid: Fundación Juan March / Sociedad Española de Literatura General y Comparada, pp. 109-116.

Yudin, Florence L. (1968). «The Novela Corta as Comedia: Lope’s Las fortunas de Diana». Bulletin of Hispanic Studies, 45, pp. 181-188.

(1969). «Theory and Practice of the Novela Comediesca». Romanische Forschungen 81, pp. 585-594.

\section{Alonso de CAStillo SolóRzano ReEscritor de sí Mismo: \\ ALGUNAS NOTAS SOBRE LOS ESCARMIENTOS DE AMOR MORALIZADOS Y EL LISARDO ENAMORADO}

RESUMEN: El presente artículo estudia la relación entre la novela Lisardo enamorado (Valencia, 1629) y su primera redacción, Escarmientos de amor moralizados (Sevilla, 1628), y las modalidades de escritura empleadas por Alonso de Castillo Solórzano en su proceso de reelaboración.

Palabras Clave: Alonso de Castillo Solórzano, Lisardo enamorado, Escarmientos de amor moralizados.

\section{Alonso de Castillo Solórzano Rewriter of Himself: \\ NOTES ON ESCARMIENTOS DE AMOR MORALIZADOS AND LISARDO ENAMORADO}

ABSTRACT: The article studies the relation between the novel Lisardo enamorado (Valencia, 1629) and its first redaction, Escarmientos de amor moralizados (Seville, 1628) and analyses the techniques of writing used by Alonso de Castillo Solórzano in the re-elaboration process.

Keywords: Alonso de Castillo Solórzano, Lisardo enamorado, Escarmientos de amor moralizados. 
Evangelina Rodríguez Cuadros (Universitat de València)

Novela cortesana, novela barroca, novela corta: de la incertidumbre al canon .9

Mita Valvassori (Universidad de Los Lagos)

El modelo narrativo del Decamerón en la Edad de Oro: una vieja historia .21

Antonio Gargano (Università degli Studi di Napoli Federico II)

«Difficile est proprie communia dicere»: el género de la novella entre

Boccaccio y Cervantes

Guillermo Carrascón (Università degli Studi di Torino)

Apuntes para un estudio de la presencia de Bandello en la

novela corta del siglo XVII

Leonardo Coppola (Università degli Studi «G. d'Annunzio» di Chieti-Pescara)

La proyección de Straparola en la novela española del Siglo de Oro desde una perspectiva editorial

Mireia Aldomì García

Didactismo, género literario y lector en Giraldi Cinzio.

María Jesús Zamora (Universidad Autónoma de Madrid)

«...En tiempo menos discreto que el de agora, aunque de hombres más sabios, se

Ilamaban a las novelas cuentos». La novela corta y el cuento en el Siglo de Oro.....109

Marcial Rubio (Università degli Studi «G. d'Annunzio» di Chieti-Pescara)

La contribución de Cervantes a la novela barroca: la ejemplaridad. .125

PIERRe Darnis (Université Bordeaux Montaigne)

La fuerza de la sangre, La ilustre fregona $y$ Las dos doncellas: ¿tres tipos

folclóricos?

María Soledad ArRedondo (Universidad Complutense de Madrid)

De La gitanilla $a$ La sabia Flora malsabidilla. El género, el personaje

y el matrimonio

Antonella Gallo (Università degli Studi di Verona)

Fabulaciones en equívocos burlescos: la Chrónica del monstro imaginado (1615)

de Alonso de Ledesma y novela corta barroca

David GonZález Ramírez (Universidad de Málaga)

El filósofo del aldea (1625) de Baltasar Mateo Velázquez: recepción textual

e hipótesis autorial.

Jonathan BRAdBury (University of Exeter)

La narrativa breve en la miscelánea del siglo XVII 
Cristina Castillo Martínez (Universidad de Jaén)

«La fuente del desengaño»: de las Noches de invierno de Eslava a la Tercera

Diana de Tejeda.

María Zerari (Université Paris-Sorbonne, CLEA)

Furor in fabula: La cruel aragonesa de Castillo Solórzano (o de la dama monstruo).. 241

Giulia Giorgi (Università degli Studi di Ferrara)

Alonso de Castillo Solórzano reescritor de sí mismo: algunas notas sobre los

Escarmientos de amor moralizados y el Lisardo enamorado .257

Angela Fabris (Alpen-Adria-Universität Klagenfurt)

El diálogo con el público y los espacios reales y de maravilla en

Casos prodigiosos y cueva encantada de Juan de Piña .267

María Rocío LePe García (IES San Sebastián, Huelva)

La traducción inglesa de Hipólito y Aminta: una adaptación

con fines comerciales 281

Andrea Bresadola (Università degli Studi di Udine)

La novela española en la Italia del siglo XVII: el caso de Il Feniso

de Francisco de Quintana

José Teruel (Universidad Autónoma de Madrid)

El triunfo del Desengaño. Marco y desengaño postrero de la Parte segunda

del Sarao y entretenimiento honesto, de María de Zayas

Nieves Romero-Díaz (Mount Holyoke College)

Lecturas alternativas en la Novela del fin bueno en mal principio

de doña Ana Francisca Abarca de Bolea.

Shifra Armon (University of Florida)

Compromiso y distanciamiento en La Venus de Ferrara

de Mariana de Carvajal Saavedra

Mechthild Albert (Rheinische Friedrich-Wilhelms-Universität Bonn)

Las "noches": un subgénero novelístico en perspectiva comparada.... .365

Fernando Copello Jouanchin (Université du Maine, Le Mans)

El mueble en la novela corta del Siglo de Oro: algunas reflexiones

en torno a la cama

Ilaria Resta (Università del Salento):

De la novella al entremés pasando por la novela corta: reescrituras del cuento

La gara delle tre mogli del Cieco di Ferrara. 


\section{EDAD DE ORO}

Revista de Filología Hispánica XXXIII

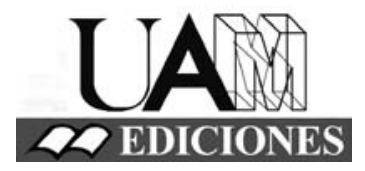




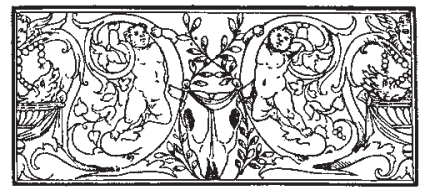

\section{Edad de Oro. Revista de Filología Hispánica}

ISSN: 0212-0429

Dirección:

Teodosio Fernández

Secretaría y edición:

José Ramón Trujillo

Coordinador del volumen XXXIII:

Rafael Bonilla Cerezo

Comité científico internacional:

Carlos Alvar (Univ. de Ginebra)

Ignacio Arellano (Univ. de Navarra)

Javier Blasco (Univ. de Valladolid)

Alberto Blecua (UAB)

Jean Canavaggio (Univ. de París X)

Laura Dolfi (Univ. de Turín)

Aurora Egido (Univ. de Zaragoza)

Víctor García de la Concha (RAE)

Luciano García Lorenzo (CSIC)

Joaquín González Cuenca (Univ. de Castilla-

La Mancha)

Agustín de La Granja (Univ. de Granada)

Begoña López Bueno (Univ. de Sevilla)

Michel Moner (Univ. de Toulouse III)

Joan Oleza (Univ. de Valencia)

Alfonso Rey (Univ. de Santiago)

Lina Rodríguez Cacho (Univ. de Salamanca)

Leonardo Romero Tobar (Univ. de Zaragoza)

Aldo Ruffinatto (Univ. de Turín)

Lía Schwartz (City University of New York)
Redacción y admisión de originales:

Teodosio Fernández

Edad de Oro

Departamento de Filología Española

Universidad Autónoma de Madrid

28049 Madrid (España)

Tfno.: +0034 914974090

correo: teodosio.fernandez@uam.es

Distribución, suscripción y venta:

Servicio de Publicaciones de la UAM

Universidad Autónoma de Madrid

28049 Madrid (España)

Intercambio de publicaciones:

Biblioteca de la Facultad de Filosofía y

Letras (UAM)

Universidad Autónoma de Madrid

28049 Madrid (España)

Han colaborado en este volumen:

Departamento de Filología Española (UAM)

Facultad de Filosofía y Letras (UAM)

Proyecto I+D FFI2013-41264-P La novela

corta del siglo XVII: estudio y edición (y II)

Edad de Oro se recoge en las siguientes bases de datos: SCOPUS, MLA Database, HLAS, Latindex, PIO-Periodical Content Index, ISOC, Dialnet, MIAR, ERIH, DICE, Sumaris CBUC, Ulrich's. Se encuentra evaluada en CIRC: A; INRECH: primer cuartil, posición 6 de 50; MIAR difusión ICDS live: 9.977; SCImago Journal \& Country Rank: H Index 2, SJR 0,101, Q4; RESH índice de impacto: 0.162; ERIH: A INT1; Carhus Plus+: B. 